\title{
ON THE GROUND STATE OF SPIN-1 BOSE-EINSTEIN CONDENSATES WITH AN EXTERNAL IOFFE-PITCHARD MAGNETIC FIELD
}

\author{
WEI LUO, ZHONGXUE LÜ® ${ }^{凶}$ and ZUHAN LIU
}

(Received 15 October 2011)

\begin{abstract}
In this paper, we prove the existence of the ground state for the spinor Bose-Einstein condensates with an external Ioffe-Pitchard magnetic field in the one-dimensional case. We also characterise the ground states of spin-1 Bose-Einstein condensates with an external Ioffe-Pitchard magnetic field; that is, for ferromagnetic systems, we show that, under some condition, searching for the ground state of ferromagnetic spin-1 Bose-Einstein condensates with an external Ioffe-Pitchard magnetic field can be reduced to a 'one-component' minimisation problem.
\end{abstract}

2010 Mathematics subject classification: primary 35J65; secondary 35B40, 35B45.

Keywords and phrases: ground states, spin-1 Bose-Einstein condensate, the external Ioffe-Pitchard magnetic field.

\section{Introduction}

The experimental realisation of Bose-Einstein condensates (BEC) in magnetically trapped atomic gases at ultra-low temperatures $[1,4,8]$ has spurred great excitement in the atomic physics community and renewed interest in studying the macroscopic quantum behaviour of atoms. In earlier BEC experiments, the atoms were confined in a magnetic trap, in which the spin degree of freedom is frozen. The particles are described by a scalar model and the wave function of the particles is governed by the Gross-Pitaevskii equation within the mean-field approximation [9, 15, 16]. One of the most important recent developments in BEC was the study of spin-1 and spin-2 condensates. In contrast to a single component BEC, a spin-F BEC is described by the coupled Gross-Pitaevskii equations which consist of $2 F+1$ equations, each governing one of the $2 F+1$ hyperfine states $\left(m_{F}=-F,-F+1, \ldots, F+1, F\right)$ within the meanfield approximation $[10,14]$. The spin-1 BEC was realised in experiments recently

Research is supported by the Natural Science Foundation of China (No. 10771181; 11071206), NSF of Jiangsu Province (No. BK2010172), the Postgraduate Innovation Project of Jiangsu Province (No. CXZZ11-0901) and Foundation of Xuzhou Normal University (No. 2011YLA003).

(C) 2012 Australian Mathematical Publishing Association Inc. 0004-9727/2012 \$16.00 
by using both ${ }^{23} \mathrm{Na}$ and ${ }^{87} \mathrm{Rb}[13,17]$. In fact, the emergence of spin-1 BEC has created great opportunities for understanding degenerate gases with internal degrees of freedom $[2,3,5,7,10,11]$.

In this paper, we consider a spin-1 BEC. For temperatures well below the critical temperature, the dynamics of the spin- $1 \mathrm{BEC}$ are well described by the dimensionless Gross-Pitaevskii equations in $n$ dimensions $(n \leqslant 3)[2,3,10]$

$$
\begin{gathered}
i \partial_{t} \psi_{1}(x, t)=\left(-\frac{1}{2} \Delta+V(x)+E_{1}+\beta_{n} \rho+\beta_{s}\left(\rho_{1}+\rho_{0}-\rho_{-1}\right)\right) \psi_{1}+\beta_{s} \bar{\psi}_{-1} \psi_{0}^{2}+B \psi_{0}, \\
i \partial_{t} \psi_{0}(x, t)=\left(-\frac{1}{2} \Delta+V(x)+E_{0}+\beta_{n} \rho+\beta_{s}\left(\rho_{1}+\rho_{-1}\right)\right) \psi_{0} \\
\quad+2 \beta_{s} \psi_{-1} \bar{\psi}_{0} \psi_{1}+B\left(\psi_{1}+\psi_{-1}\right) \\
i \partial_{t} \psi_{-1}(x, t)=\left(\begin{array}{rl}
\frac{1}{2} \Delta & \left.+V(x)+E_{-1}+\beta_{n} \rho+\beta_{s}\left(\rho_{-1}+\rho_{0}-\rho_{1}\right)\right) \psi_{-1} \\
& +\beta_{s} \bar{\psi}_{1} \psi_{0}^{2}+B \psi_{0}
\end{array}\right.
\end{gathered}
$$

where $x \in \mathbb{R}^{n}, t>0$, and the initial value $(t=0)$

$$
\psi_{j}(x, 0)=\psi_{j}^{0}(x), \quad x \in \mathbb{R}^{n}, j=-1,0,1 .
$$

Here, $\Psi=\Psi(x, t):=\left(\psi_{1}(x, t), \psi_{0}(x, t), \psi_{-1}(x, t)\right)^{T}$ is the dimensionless wave function of the spin-1 BEC, $V(x)$ is the dimensionless external trapping potential, $\rho_{j}(x, t):=$ $\left|\psi_{j}(x, t)\right|^{2}$ is the density of the hyperfine spin component $m_{F}=j(j=-1,0,1)$ and $\rho=\rho_{1}+\rho_{0}+\rho_{-1}$ is the total density. Also, $E_{j} \in \mathbb{R}$ is the dimensionless Zeeman energy of spin component $m_{F}=j(j=-1,0,1)$ in the uniform external magnetic field, $B \in \mathbb{R}$ is the dimensionless external Ioffe-Pitchard magnetic field, and $\beta_{n}$ and $\beta_{s}$ are the dimensionless mean-field and spin-exchange interaction constants, respectively. Furthermore, $\bar{f}$ denotes the conjugate of the function $f$. For $\beta_{n}<0$ (respectively $\beta_{n}>0$ ) the spin-independent interaction is attractive (respectively repulsive). For $\beta_{s}<0$ (respectively $\beta_{s}>0$ ), the spin-exchange interaction is ferromagnetic (respectively anti-ferromagnetic).

For the ferromagnetic system (1.1)-(1.3) with $n=1$, Cao et al. [6] proved the existence of the ground state without the Ioffe-Pitchard magnetic field. Recently, Lin et al. [12] characterised the ground states of spin-1 Bose-Einstein condensates under no external magnetic field. Motivated by [6, 12], the aim of this paper is to study the ground state of the ferromagnetic system (1.1)-(1.3) for $n=1$. We consider the simplest case when $V(x) \equiv 0$ and all $\psi_{j}(j=-1,0,1)$ are real. We rename $\psi_{j}$ by $u_{j}(j=-1,0,1)$. From (1.1)-(1.3), the energy functional is

$$
\begin{aligned}
H\left(u_{-1}, u_{0}, u_{1}\right)= & \int_{\mathbb{R}} \\
& \left(\sum_{j=-1}^{1}\left(\frac{1}{2}\left|u_{j}^{\prime}\right|^{2}+E_{j}\right) u_{j}^{2}+\frac{\beta_{n}}{2} u_{0}^{4}+\frac{\beta_{n}+\beta_{s}}{2}\left(u_{1}^{4}+u_{-1}^{4}+2 u_{0}^{2}\left(u_{1}^{2}+u_{-1}^{2}\right)\right)\right. \\
& \left.+\left(\beta_{n}-\beta_{s}\right) u_{1}^{2} u_{-1}^{2}+2 \beta_{s} u_{1} u_{0}^{2} u_{-1}+2 B u_{0}\left(u_{1}+u_{-1}\right)\right) d x
\end{aligned}
$$


and the following two integrals are conserved:

$$
\begin{gathered}
\int_{\mathbb{R}} \sum_{j=-1}^{1} u_{j}^{2}(x) d x=N, \\
\int_{\mathbb{R}}\left(u_{1}^{2}(x)-u_{-1}^{2}(x)\right) d x=M .
\end{gathered}
$$

Here we assume that

$$
N>0, \quad|M|<N .
$$

For given real numbers $(N, M)$, we define

$$
\begin{aligned}
E_{N, M}=\{u & =\left(u_{-1}, u_{0}, u_{1}\right) \mid u_{j} \in H^{1}(\mathbb{R}), j=1,2,3, \\
& \left.\int_{\mathbb{R}} \sum_{j=-1}^{1} u_{j}^{2}(x) d x=N, \int_{\mathbb{R}}\left(u_{1}^{2}(x)-u_{-1}^{2}(x)\right) d x=M\right\} .
\end{aligned}
$$

We consider the minimisation problem

$$
H_{0}=\inf \left\{H(u) \mid u \in E_{N, M}\right\} .
$$

A solution to (1.6) is called a ground state. A ground state $\left(u_{-1}, u_{0}, u_{1}\right)$ is nontrivial if $u_{j} \neq 0$, for $j=-1,0,1$.

Our main result in this paper is the following theorem.

Theorem 1.1. Let $\beta_{n}<\beta_{s}<0$ and $E_{0}=E_{-1} \leq E_{1}<0, B<0, \min \left\{\left|E_{j}\right|, j=-1,0,1\right\}>$ $2|B|$. Then a nontrivial ground state exists. Moreover, the ground state $\left(u_{-1}, u_{0}, u_{1}\right)$ is positive and strictly decreasing.

Following the method in [6], Theorem 1.1 is proved via approximation. Namely, we consider a related minimisation problem in a bounded interval $I_{k}:=[-k, k]$ and then let $k \rightarrow+\infty$. More precisely, let us define an energy functional on $I_{k}$ :

$$
\begin{aligned}
H^{k}\left(u_{-1}, u_{0}, u_{1}\right)=\int_{I_{k}} & \left(\sum_{j=-1}^{1}\left(\frac{1}{2}\left|u_{j}^{\prime}\right|^{2}+E_{j}\right) u_{j}^{2}+\frac{\beta_{n}}{2} u_{0}^{4}+\frac{\beta_{n}+\beta_{s}}{2}\left(u_{1}^{4}+u_{-1}^{4}+2 u_{0}^{2}\left(u_{1}^{2}+u_{-1}^{2}\right)\right)\right. \\
& \left.+\left(\beta_{n}-\beta_{s}\right) u_{1}^{2} u_{-1}^{2}+2 \beta_{s} u_{1} u_{0}^{2} u_{-1}+2 B u_{0}\left(u_{1}+u_{-1}\right)\right) d x
\end{aligned}
$$

For given real numbers $(N, M)$, we define

$$
\begin{aligned}
E_{N, M}^{k}=\{u & =\left(u_{-1}, u_{0}, u_{1}\right) \mid u_{j} \in H_{0}^{1}\left(I_{k}\right), j=1,2,3, \\
& \left.\int_{I_{k}} \sum_{j=-1}^{1} u_{j}^{2}(x) d x=N, \int_{I_{k}}\left(u_{1}^{2}(x)-u_{-1}^{2}(x)\right) d x=M\right\} .
\end{aligned}
$$


We consider the minimisation problem

$$
H_{0}^{k}=\inf \left\{H^{k}(u) \mid u \in E_{N, M}^{k}\right\} .
$$

It is easy to see that

$$
H_{0}^{k} \rightarrow H_{0} \quad \text { as } k \rightarrow \infty
$$

We will prove the following theorem.

Theorem 1.2. Let $\beta_{n}<\beta_{s}<0$ and $E_{0}=E_{-1} \leq E_{1}<0, B<0$. Then the minimisation problem (1.8) can be attained by some $u^{k}=\left(u_{-1, k}, u_{0, k}, u_{1, k}\right)$ where $u_{j, k}>0$ and are strictly decreasing.

Furthermore, in the last section, we will characterise the ground states of spin-1 Bose-Einstein condensates with an external Ioffe-Pitchard magnetic field (1.1)-(1.3).

We organise this paper as follows. In Section 2, we give the proof of Theorem 1.2. In Section 3, we prove Theorem 1.1. In Section 4, following the method in [12] by Lin et al., we characterise the ground state.

\section{Proof of Theorem 1.2}

We now prove Theorem 1.2.

We rewrite $H^{k}$ as follows:

$$
\begin{aligned}
H^{k}\left(u_{-1}, u_{0}, u_{1}\right)=\int_{I_{k}} & \left(\sum_{j=-1}^{1}\left(\frac{1}{2}\left|u_{j}^{\prime}\right|^{2}+E_{j} u_{j}^{2}\right)+\frac{\beta_{n}}{2}\left(\sum_{j=-1}^{1} u_{j}^{2}\right)^{2}\right) d x \\
& +\frac{\beta_{s}}{2} \int_{I_{k}}\left(\left(u_{1}^{2}-u_{-1}^{2}\right)^{2}+2 u_{0}^{2}\left(u_{1}+u_{-1}\right)^{2}\right) d x \\
& +\int_{I_{k}} 2 B u_{0}\left(u_{1}+u_{-1}\right) d x
\end{aligned}
$$

Let $u^{l}=\left(u_{-1}^{l}, u_{0}^{l}, u_{1}^{l}\right)$ be a minimising sequence of (1.8). We can always assume that each component $u_{j}^{l}$ is nonnegative, since it is easy to see that

$$
H^{k}\left(\left|u_{-1}^{l}\right|,\left|u_{0}^{l}\right|,\left|u_{1}^{l}\right|\right) \leq H^{k}\left(u_{-1}^{l}, u_{0}^{l}, u_{1}^{l}\right)
$$

and $\left(\left|u_{-1}^{l}\right|,\left|u_{0}^{l}\right|,\left|u_{1}^{l}\right|\right) \in E_{N, M}^{k}$. Hence we can replace $\left(u_{-1}^{l}, u_{0}^{l}, u_{1}^{l}\right)$ by $\left(\left|u_{-1}^{l}\right|,\left|u_{0}^{l}\right|,\left|u_{1}^{l}\right|\right)$.

For $u \in H^{1}(\mathbb{R}), u \geq 0$, let us denote its Schwarz symmetrisation by $u^{*}$. Then (see [17])

$$
\begin{gathered}
\int_{\mathbb{R}}\left|u_{j}^{\prime}\right|^{2} d x \geq \int_{\mathbb{R}}\left|\left(u_{j}^{*}\right)^{\prime}\right|^{2} d x, \quad j=-1,0,1, \\
\int_{\mathbb{R}} u_{j}^{2} d x=\int_{\mathbb{R}}\left(u_{j}^{*}\right)^{2} d x, \quad \int_{\mathbb{R}} u_{j}^{4} d x=\int_{\mathbb{R}}\left(u_{j}^{*}\right)^{4} d x, \quad j=-1,0,1,
\end{gathered}
$$




$$
\begin{gathered}
\int_{\mathbb{R}} u_{j} u_{k} d x=\int_{\mathbb{R}}\left(u_{j}^{*}\right)\left(u_{k}^{*}\right) d x, \quad \int_{\mathbb{R}} u_{j}^{2} u_{k}^{2} d x=\int_{\mathbb{R}}\left(u_{j}^{*}\right)^{2}\left(u_{k}^{*}\right)^{2} d x, \quad j=-1,0,1, \\
\int_{\mathbb{R}} u_{j} u_{k} d x=\int_{\mathbb{R}}\left(u_{j}^{*}\right)\left(u_{k}^{*}\right) d x, \quad \int_{\mathbb{R}} u_{-1} u_{1} u_{0}^{2} d x=\int_{\mathbb{R}} u_{-1}^{*} u_{1}^{*}\left(u_{0}^{*}\right)^{2} d x, \quad j=-1,0,1,
\end{gathered}
$$

which imply that

$$
H^{k}\left(\left(u_{-1}^{l}\right)^{*},\left(u_{0}^{l}\right)^{*},\left(u_{1}^{l}\right)^{*}\right) \leq H^{k}\left(u_{-1}^{l}, u_{0}^{l}, u_{1}^{l}\right)
$$

and $\left(\left(u_{-1}^{l}\right)^{*},\left(u_{0}^{l}\right)^{*},\left(u_{1}^{l}\right)^{*}\right) \in E_{N, M}^{k}$.

Now we can assume that $u_{j}^{l}$ are nonnegative, even and nonincreasing in $I_{k}$. Next we show that the minimising sequence is uniformly bounded in $H_{0}^{1}\left(I_{k}\right)$ for $k \geq 1$.

By the Gagliardo-Nirenberg inequality [2] and Young's inequality, for any $u \in$ $H_{0}^{1}\left(I_{k}\right)$, we have that, for any $\varepsilon>0$, there exists $C(\varepsilon)>0$ such that

$$
\int_{I_{k}}|u|^{4} d x \leq C \varepsilon \int_{I_{k}}\left|u^{\prime}\right|^{2} d x+C(\varepsilon)\left(\int_{I_{k}}|u|^{2} d x\right)^{3} .
$$

Then by the Cauchy inequality and Young's inequality and (2.1),

$$
\begin{gathered}
\int_{I_{k}} u_{k} u_{j} d x \leq \frac{1}{2} \int_{I_{k}}\left(u_{k}^{2}+u_{j}^{2}\right) d x \leq \frac{1}{2} N \\
\int_{I_{k}} u_{k}^{2} u_{j}^{2} d x \leq C \varepsilon \int_{I_{k}}\left(\left|u_{k}^{\prime}\right|^{2}+\left|u_{j}^{\prime}\right|^{2}\right) d x+C(\varepsilon)\left(\left(\int_{I_{k}} u_{k}^{2} d x\right)^{3}+\left(\int_{I_{k}} u_{j}^{2} d x\right)^{3}\right),
\end{gathered}
$$

and

$$
\begin{aligned}
\int_{I_{k}} u_{l}^{2} u_{k} u_{j} d x \leq C \varepsilon & \int_{I_{k}}\left(\left|u_{l}^{\prime}\right|^{2}+\left|u_{k}^{\prime}\right|^{2}+\left|u_{j}^{\prime}\right|^{2}\right) d x \\
& +C(\varepsilon)\left(\left(\int_{I_{k}} u_{l}^{2} d x\right)^{3}+\left(\int_{I_{k}} u_{k}^{2} d x\right)^{3}+\left(\int_{I_{k}} u_{j}^{2} d x\right)^{3}\right)
\end{aligned}
$$

Hence,

$$
\begin{aligned}
\frac{1}{2} \int_{I_{k}} \sum_{j=-1}^{1}\left|\left(u_{j}^{l}\right)^{\prime}\right|^{2} d x= & H_{0}^{k}-\int_{I_{k}} \sum_{j=-1}^{1} E_{j}\left(u_{j}^{l}\right)^{2} d x-\frac{\beta_{n}}{2}\left(\sum_{j=-1}^{1}\left(u_{j}^{l}\right)^{2}\right)^{2} d x \\
& -\frac{\beta_{s}}{2} \int_{I_{k}}\left(\left(\left(u_{1}^{l}\right)^{2}-\left(u_{-1}^{l}\right)^{2}\right)^{2}+2\left(u_{0}^{l}\right)^{2}\left(u_{1}^{l}+u_{-1}^{l}\right)^{2}\right) d x \\
& -2 B \int_{I_{k}} u_{0}^{l}\left(u_{1}^{l}+u_{-1}^{l}\right) d x+o_{l}(1) \\
\leq & H_{0}^{k}-\frac{C \varepsilon\left(\beta_{n}+\beta_{s}\right)}{2} \int_{I_{k}} \sum_{j=-1}^{1}\left|\left(u_{j}^{l}\right)^{\prime}\right|^{2} d x+C(\varepsilon) N^{3}-2 B N
\end{aligned}
$$


Choosing $\varepsilon$ sufficiently small, we have

$$
\int_{I_{k}}\left(\sum_{j=-1}^{1}\left|\left(u_{j}^{l}\right)^{\prime}\right|^{2}+\left(u_{j}^{l}\right)^{2}\right) d x<C
$$

which implies that by Sobolev embedding

$$
\left\|u_{j}^{l}\right\|_{L^{\infty}}<C, \quad j=-1,0,1, l=1,2, \ldots
$$

Then we can obtain the existence of the minimiser $\left(u_{-1, k}, u_{0, k}, u_{1, k}\right)$ by applying compactness of the embedding of the subspace of $H_{0}^{1}\left(I_{k}\right)$ that consists of even functions into $L^{4}\left(I_{k}\right)$. We can also assume that $u_{j, k} \geq 0$, for $j=-1,0,1$, and the least component is not identically 0 .

Furthermore, there are Lagrange multipliers $\lambda^{k}, \mu^{k}$ such that $\left(u_{-1, k}, u_{0, k}, u_{1, k}\right)$ satisfies the Euler-Lagrange equations

$$
\left\{\begin{aligned}
\begin{array}{rl}
\frac{1}{2} u_{-1}^{\prime \prime} & -E_{-1} u_{-1}-\left(\beta_{n}+\beta_{s}\right) u_{-1}^{3}-\left(\left(\beta_{n}+\beta_{s}\right) u_{0}^{2}+\left(\beta_{n}-\beta_{s}\right) u_{1}^{2}\right) u_{-1}-\beta_{s} u_{0}^{2} u_{1}-B u_{0} \\
& =\left(\lambda^{k}-\mu^{k}\right) u_{-1} \quad \text { in } I_{k},
\end{array} \\
\begin{array}{rl}
\frac{1}{2} u_{0}^{\prime \prime}- & E_{0} u_{0}-\beta_{n} u_{0}^{3}-\left(\beta_{n}+\beta_{s}\right)\left(u_{1}^{2}+u_{-1}^{2}\right) u_{0}-2 \beta_{s} u_{-1} u_{0} u_{1}-B\left(u_{1}+u_{-1}\right) \\
& =\lambda^{k} u_{0} \quad \text { in } I_{k},
\end{array} \\
\frac{1}{2} u_{1}^{\prime \prime}-E_{1} u_{1}-\left(\beta_{n}+\beta_{s}\right) u_{1}^{3}-\left(\beta_{n}+\beta_{s}\right) u_{0}^{2} u_{1}-\left(\beta_{n}-\beta_{s}\right) u_{-1}^{2} u_{1}-\beta_{s} u_{0}^{2} u_{-1}-B u_{0} \\
\quad=\left(\lambda^{k}+\mu^{k}\right) u_{1} \quad \text { in } I_{k}, \\
u_{j}>0 \text { in }(-k, k), \quad u_{j}( \pm k)=0, j=-1,0,1 .
\end{aligned}\right.
$$

In what follows, we show that $u_{j}(k) \neq 0$ for $j=-1,0,1$. This will be done by two claims.

\section{Claim 1. $u_{0, k}>0$.}

We argue by contradiction. Suppose that $u_{0, k} \geq 0$ and $u_{0, k}\left(x_{0}\right)=0$. Then by the maximum principle, $u_{0, k} \equiv 0$. Hence $\left(u_{-1, k}, u_{1, k}\right)$ is a solution of

$$
\left\{\begin{array}{l}
\frac{1}{2} u_{-1}^{\prime \prime}-E_{-1} u_{-1}-\left(\beta_{n}+\beta_{s}\right) u_{-1}^{3}-\left(\beta_{n}-\beta_{s}\right) u_{1}^{2} u_{-1}=\left(\lambda^{k}-\mu^{k}\right) u_{-1} \quad \text { in } I_{k}, \\
\frac{1}{2} u_{1}^{\prime \prime}-E_{1} u_{1}-\left(\beta_{n}+\beta_{s}\right) u_{1}^{3}-\left(\beta_{n}-\beta_{s}\right) u_{-1}^{2} u_{1}=\left(\lambda^{k}+\mu^{k}\right) u_{1} \quad \text { in } I_{k}
\end{array}\right.
$$

satisfying the constraint,

$$
\int_{I_{k}} u_{1, k}^{2} d x=\frac{N+M}{2}, \quad \int_{I_{k}} u_{-1, k}^{2} d x=\frac{N-M}{2} .
$$

Set $\left(u_{-1}, u_{0}, u_{1}\right)=\left(u_{-1, k}+\varepsilon_{-1} \psi_{-1}, \varepsilon_{0} \psi_{0}, u_{1, k}+\varepsilon_{1} \psi_{1}\right)$ with $\varepsilon_{j}>0, \psi_{j} \in H_{0}^{1}\left(I_{k}\right)$ for $j=-1,0,1$ such that

$$
\begin{gathered}
\int_{I_{k}}\left(\left|u_{-1, k}+\varepsilon_{-1} \psi_{-1}\right|^{2}+\left|\varepsilon_{0} \psi_{0}\right|^{2}+\left|u_{1, k}+\varepsilon_{1} \psi_{1}\right|^{2}\right) d x=N, \\
\int_{I_{k}}\left(\left|u_{1, k}+\varepsilon_{1} \psi_{1}\right|^{2}-\left|u_{-1, k}+\varepsilon_{-1} \psi_{-1}\right|^{2}\right) d x=M .
\end{gathered}
$$


This can be done by choosing $\varepsilon_{j}>0, \psi_{j} \in H_{0}^{1}\left(I_{k}\right), j=-1,0,1$ such that $\int_{I_{k}} u_{-1, k} \psi_{-1} d x$ $<0, \int_{I_{k}} u_{1, k} \psi_{1} d x<0$ and

$$
\begin{gathered}
2 \varepsilon_{1} \int_{I_{k}} u_{1, k} \psi_{1} d x+\varepsilon_{1}^{2} \int_{I_{k}}\left|\psi_{1}\right|^{2} d x=2 \varepsilon_{-1} \int_{I_{k}} u_{-1, k} \psi_{-1} d x+\varepsilon_{-1}^{2} \int_{I_{k}}\left|\psi_{-1}\right|^{2} d x \\
2 \varepsilon_{1} \int_{I_{k}} u_{1, k} \psi_{1} d x+\varepsilon_{1}^{2} \int_{I_{k}}\left|\psi_{1}\right|^{2} d x+2 \varepsilon_{-1} \int_{I_{k}} u_{-1, k} \psi_{-1} d x \\
+\varepsilon_{-1}^{2} \int_{I_{k}}\left|\psi_{-1}\right|^{2} d x+\varepsilon_{0}^{2} \int_{I_{k}}\left|\psi_{0}\right|^{2} d x=0 .
\end{gathered}
$$

Then

$$
\begin{gathered}
\varepsilon_{1} \int_{I_{k}} u_{1, k} \psi_{1} d x=\varepsilon_{-1} \int_{I_{k}} u_{-1, k} \psi_{-1} d x+O\left(\varepsilon_{-1}^{2}+\varepsilon_{1}^{2}\right) \\
\varepsilon_{1} \int_{I_{k}} u_{1, k} \psi_{1} d x+\varepsilon_{-1} \int_{I_{k}} u_{-1, k} \psi_{-1} d x=-\frac{1}{2} \varepsilon_{0}^{2} \int_{I_{k}}\left|\psi_{0}\right|^{2} d x+O\left(\varepsilon_{-1}^{2}+\varepsilon_{1}^{2}\right) .
\end{gathered}
$$

Applying the above equality to the expression in $E^{k}\left(u_{-1}, u_{0}, u_{1}\right)$, we have

$$
\begin{aligned}
E^{k}\left(u_{-1}, u_{0}, u_{1}\right)=- & 2 \varepsilon_{1}\left(\lambda^{k}+\mu^{k}\right) \int_{I_{k}} u_{1, k} \psi_{1} d x-2 \varepsilon_{-1}\left(\lambda^{k}-\mu^{k}\right) \int_{I_{k}} u_{-1, k} \psi_{-1} d x \\
& +\varepsilon_{0}^{2} \int_{I_{k}}\left(\frac{1}{2}\left(\psi_{0}^{\prime}\right)^{2}+E_{0} \psi_{0}^{2}+\left(\beta_{n}+\beta_{s}\right)\left(u_{1, k}^{2}+u_{-1, k}^{2}\right) \psi_{0}^{2}\right. \\
& \left.+2 \beta_{s} u_{1, k} u_{-1, k} \psi_{0}^{2}\right) d x+E^{k}\left(u_{-1, k}, 0, u_{1, k}\right)+O\left(\varepsilon_{-1}^{4}+\varepsilon_{1}^{4}\right) \\
=\varepsilon_{0}^{2} & \int_{I_{k}}\left(\frac{1}{2}\left|\psi_{0}^{\prime}\right|^{2}+\left(E_{0}+\lambda^{k}\right) \psi_{0}^{2}+\left(\beta_{n}+\beta_{s}\right)\left(u_{1, k}^{2}+u_{-1, k}^{2}\right) \psi_{0}^{2}\right. \\
& \left.+2 \beta_{s} u_{1, k} u_{-1, k} \psi_{0}^{2}\right) d x+E^{k}\left(u_{-1, k}, 0, u_{1, k}\right)+O\left(\varepsilon_{-1}^{2}+\varepsilon_{1}^{2}\right) \\
& +O\left(\varepsilon_{-1}^{4}+\varepsilon_{1}^{4}\right) .
\end{aligned}
$$

By (2.2) satisfied by $u_{-1, k}, u_{1, k}$,

$$
\begin{aligned}
& \int_{I_{k}}\left(\frac{1}{2}\left(\left|u_{1, k}^{\prime}\right|^{2}+\eta^{2}\left|u_{-1, k}^{\prime}\right|^{2}\right)+E_{1} u_{1, k}^{2}+\eta^{2} E_{-1}\left|u_{-1, k}^{\prime}\right|^{2}+\left(\beta_{n}+\beta_{s}\right)\left(u_{1, k}^{4}+\eta^{2} u_{-1, k}^{4}\right)\right. \\
& \left.\quad+\left(\beta_{n}-\beta_{s}\right)\left(1+\eta^{2}\right) u_{-1, k}^{2} u_{1, k}^{2}\right) d x \\
& \quad=-\left(\lambda^{k}+\mu^{k}\right) \int_{I_{k}} u_{1, k}^{2} d x-\left(\lambda^{k}-\mu^{k}\right) \int_{I_{k}} \eta^{2} u_{-1, k}^{2} d x
\end{aligned}
$$

By (2.2) satisfied by $u_{-1, k}, u_{1, k}$ again,

$$
\begin{gathered}
\int_{I_{k}}\left(u_{1, k}^{\prime} u_{-1, k}^{\prime}+\left(E_{1}+E_{-1}\right) u_{1, k} u_{-1, k}+2 \beta_{n}\left(u_{1, k}^{3} u_{-1, k}+u_{-1, k}^{3} u_{1, k}\right)\right) d x \\
=-2 \lambda^{k} \int_{I_{k}} u_{1, k} u_{-1, k} d x .
\end{gathered}
$$


Let $\eta=((N+M) /(N-M))^{1 / 2}$. Then by $\beta_{s}<0, E_{0}=E_{-1} \leq E_{1}<0$ and from (2.4) and (2.5),

$$
\begin{aligned}
& \int_{I_{k}}\left(\frac{1}{2}\left|\left(u_{1, k}+\eta u_{-1, k}\right)^{\prime}\right|^{2}+\left(E_{0}+\lambda^{k}+\left(\beta_{n}+\beta_{s}\right)\left(u_{1, k}^{2}+u_{-1, k}^{2}\right)\right.\right. \\
& \left.\left.\quad+2 \beta_{s} u_{1, k} u_{-1, k}\right)\left(u_{1, k}+\eta u_{-1, k}\right)^{2}\right) d x<0 .
\end{aligned}
$$

Let $\psi_{0}=u_{1, k}+\eta u_{-1, k}$. Then by (2.3) and (2.6), when $\varepsilon_{-1}, \varepsilon_{0}, \varepsilon_{1}$ are sufficiently large, we have $E^{k}\left(u_{-1}, u_{0}, u_{1}\right)<E_{0}$. This is a contradiction. Hence $u_{0, k}>0$.

Claim 2. $u_{-1, k}>0, u_{1, k}>0$.

Suppose that $u_{1, k} \geq 0$ and $u_{1, k}\left(x_{0}\right)=0$. By the maximum principle, $u_{1, k}(x) \equiv 0$. By the equation satisfied by $u_{1, k}$,

$$
\beta_{s} u_{0, k}^{2} u_{-1, k}+B u_{0, k}=0 .
$$

Therefore either $u_{0, k} \equiv 0$ or $\beta_{s} u_{0, k} u_{-1, k}+B \equiv 0$. But by Claim 1 and $\beta_{s}<0, B<0$, this is impossible. Hence $u_{1, k}>0$. Similarly, we can prove $u_{-1, k}>0$. Thus we have completed the proof of Theorem 1.2.

\section{Proof of Theorem 1.1}

From Section 2, for each $k \geq 1$, we obtain a minimiser to the minimisation problem (1.7) which satisfies the following Euler-Lagrange equations

$$
\left\{\begin{array}{l}
\frac{1}{2} u_{-1, k}^{\prime \prime}-E_{-1} u_{-1, k}-\left(\beta_{n}+\beta_{s}\right) u_{-1, k}^{3}-\left[\left(\beta_{n}+\beta_{s}\right) u_{0, k}^{2}+\left(\beta_{n}-\beta_{s}\right) u_{1, k}^{2}\right] u_{-1, k} \\
\quad-\beta_{s} u_{0, k}^{2} u_{1, k}-B u_{0, k}=\left(\lambda^{k}-\mu^{k}\right) u_{-1, k} \quad \text { in }(-k, k), \\
\frac{1}{2} u_{0, k}^{\prime \prime}-E_{0} u_{0, k}-\beta_{n} u_{0, k}^{3}-\left(\beta_{n}+\beta_{s}\right)\left(u_{1, k}^{2}+u_{-1, k}^{2}\right) u_{0, k}-2 \beta_{s} u_{-1, k} u_{0, k} u_{1, k}-B\left(u_{1, k}+u_{-1, k}\right) \\
\quad=\lambda^{k} u_{0, k} \quad \text { in }(-k, k), \\
\frac{1}{2} u_{1, k}^{\prime \prime}-E_{1} u_{1, k}-\left(\beta_{n}+\beta_{s}\right) u_{1, k}^{3}-\left(\beta_{n}+\beta_{s}\right) u_{0, k}^{2} u_{1, k}-\left(\beta_{n}-\beta_{s}\right) u_{-1, k}^{2} u_{1, k} \\
\quad-\beta_{s} u_{0, k}^{2} u_{-1, k}-B u_{0, k}=\left(\lambda^{k}+\mu^{k}\right) u_{1, k} \quad \text { in }(-k, k), \\
u_{j, k}>0 \text { in }(-k, k), \quad u_{j, k}( \pm k)=0, j=-1,0,1 .
\end{array}\right.
$$

From Section 2, we also know the following results:

(1) $u_{j, k}>0$ in $(-k, k), u_{j, k}$ is even and decreasing;

(2) for $k \geq k_{0}, H_{0}^{k} \leq c_{0}<0$.

Indeed, from (1.8), we only need prove $H_{0}<0$. Set $v_{j}(x)=\rho^{1 / 2} u_{j}(\rho x)$ for $j=-1,0,1$. Then for any $\rho>0$, we have that $\left(v_{-1}, v_{0}, v_{1}\right)$ also satisfies

$$
\begin{gathered}
\int_{\mathbb{R}} \sum_{j=-1}^{1} v_{j}^{2}(x) d x=N, \\
\int_{\mathbb{R}}\left(v_{1}^{2}(x)-v_{-1}^{2}(x)\right) d x=M,
\end{gathered}
$$


and

$$
\begin{aligned}
H\left(v_{-1}, v_{0}, v_{1}\right)=\rho^{2} & \int_{\mathbb{R}} \sum_{j=-1}^{1} \frac{1}{2}\left|u_{j}^{\prime}\right|^{2} d x+\int_{\mathbb{R}} \sum_{j=-1}^{1} E_{j} u_{j}^{2} d x+\frac{\rho \beta_{n}}{2} \int_{\mathbb{R}}\left(\sum_{j=-1}^{1} u_{j}^{2}\right)^{2} d x \\
& +\frac{\rho \beta_{s}}{2} \int_{\mathbb{R}}\left(\left(u_{1}^{2}-u_{-1}^{2}\right)^{2}+2 u_{0}^{2}\left(u_{1}^{2}+u_{-1}^{2}\right)+4 u_{1} u_{0}^{2} u_{-1}\right. \\
& \left.+2 u_{0}^{2}\left(u_{1}+u_{-1}\right)^{2}\right) d x+\int_{\mathbb{R}} 2 B u_{0}\left(u_{1}+u_{-1}\right) d x
\end{aligned}
$$

Then $H_{0}<0$ follows from (3.1) and $\beta_{n}<\beta_{s}<0, E_{j}<0, B<0, \min \left\{\left|E_{j}\right|, j=-1,0,1\right\}>$ $2|B|$, by taking $\rho$ small enough.

As well as (1) and (2), we have the following result:

(3) $\left\|u_{j, k}\right\|_{H^{1}\left(I_{k}\right)} \leq C$ for some $C$.

Thus by Morrey's inequality, we can take a subsequence of $k \rightarrow \infty$ such that $u_{j, k} \rightarrow u_{j}$ uniformly in $\mathbb{R}$ where $u_{j} \in H^{1}(\mathbb{R}), u_{j} \geq 0$ and $u_{j}$ is decreasing. Then we can conclude that $u_{j, k} \rightarrow u_{j}$ in $L^{p}(\mathbb{R})$ for $p>2$. But since $u_{j, k} \rightarrow u_{j}$ uniformly in $\mathbb{R}$, we cannot conclude $u_{j, k} \rightarrow u_{j}$ in $L^{2}(\mathbb{R})$. Note that if we can prove that $u_{j, k} \rightarrow u_{j}$ in $L^{2}(\mathbb{R})$, then $\left(u_{-1}, u_{0}, u_{1}\right)$ satisfies the constraint (1.4)-(1.5) and is a minimiser of the minimisation problem (1.6). By the same arguments as for Claim 1 and Claim 2, we can prove that $u_{j}>0$.

Similarly to the proof in [6], we can prove strong convergence in $L^{2}(\mathbb{R})$. We will give the detailed proof in a few claims.

Claim 3. $\lim _{k \rightarrow+\infty}\left(\lambda^{k}-\mu^{k}\right) \geq 0, \lim _{k \rightarrow+\infty} \lambda^{k} \geq 0, \lim _{k \rightarrow+\infty}\left(\lambda^{k}+\mu^{k}\right) \geq 0$.

In fact, suppose $\lim _{k \rightarrow+\infty}\left(\lambda^{k}+\mu^{k}\right)<-c_{0}<0$. Then from the equation for $u_{1, k}$, we see that

$$
u_{1, k}^{\prime \prime}+\frac{c_{0}}{4} u_{1, k} \leq 0, \quad u_{1, k}(x)>0 \quad \text { in }(-k, k)
$$

But by the Liouville comparison theorem, for $k$ large, $u_{1, k}$ must change signs in $\left(-\sqrt{c_{0}} \pi, \sqrt{c_{0}} \pi\right)$, which is a contradiction to the fact that $u_{1, k}(x)>0$ in $(-k, k)$.

The other cases can be proven similarly.

Claim 4. There exists a positive constant $c_{0}>0$ such that

$$
\lambda^{k} N+\mu^{k} M \geq c_{0}>0 .
$$

In fact, by integrating by parts,

$$
\begin{gathered}
-\int_{I_{k}} \sum_{j=-1}^{1}\left(\frac{1}{2}\left(u_{j, k}^{\prime}\right)^{2}+E_{j} u_{j, k}^{2}\right) d x-\int_{I_{k}} \beta_{n}\left(\sum_{j=-1}^{1} u_{j, k}^{2}\right)^{2} d x \\
-\int_{I_{k}} \beta_{s}\left(\left(u_{1, k}^{2}-u_{-1, k}^{2}\right)^{2}+2 u_{0, k}^{2}\left(u_{1, k}+u_{-1, k}\right)^{2}\right) d x-\int_{I_{k}} 2 B u_{0, k}\left(u_{1, k}+u_{-1, k}\right) d x \\
=\lambda^{k} N+\mu^{k} M \geq-H^{k}\left(u_{-1, k}, u_{0, k}, u_{1, k}\right) \geq c_{0}>0
\end{gathered}
$$

for $k$ large. 
Claim 5. There exists $c_{0}>0$ such that $\lambda^{k} \geq c_{0}>0$ for $k$ large and $\int_{I_{k}} u_{0, k}^{2} d x \rightarrow \int_{\mathbb{R}} u_{0}^{2} d x$ as $k \rightarrow+\infty$.

From Claim 3, we deduce that $\lim _{k \rightarrow+\infty}\left(\lambda^{k}-\left|\mu^{k}\right|\right) \geq 0$. By Claim 4,

$$
\lambda^{k} \geq c_{0}>0
$$

In fact, if $M=0$, then (3.2) is obvious. If $M>0$, then $\lambda^{k} N+\lambda^{k} M \geq \lambda^{k} N+\mu^{k} M \geq$ $c_{0}>0$ for $k$ large. If $M<0$, then $\lambda^{k} N-\lambda^{k} M \geq \lambda^{k} N+\mu^{k} M \geq c_{0}>0$ for $k$ large.

For $\delta=\sqrt{(3 / 4) c_{0} /\left(-3 \beta_{n}-4 \beta_{s}\right)}$, we can find $R>0$ and $k_{0}$ such that

$$
u_{1, k}(x) \leq \delta, \quad u_{-1, k}(x) \leq \delta \quad \text { for }|x|>R, k \geq k_{0} .
$$

From the equation for $u_{0, k}$ and (3.3),

$$
u_{0, k}^{\prime \prime}-\frac{c_{0}}{4} u_{0, k} \geq 0 \quad \text { for }|x|>R, k \geq k_{0}
$$

where $R$ is fixed large number. By the comparison principle,

$$
u_{0, k}(x) \leq u_{0, k}(R) e^{-\sqrt{c_{0}} / 4(|x|-R)} \leq C e^{-\sqrt{c_{0}} / 4|x|} .
$$

Note that $R$ depends only on $c_{0}$. Thus we conclude that $u_{0, k}$ has exponential decay. So $\int_{I_{k}} u_{0, k}^{2} d x \rightarrow \int_{\mathbb{R}} u_{0}^{2} d x$ as $k \rightarrow \infty$.

Since $\lambda^{k} \geq c_{0}>0$, we see that either $\lambda^{k}+\mu^{k} \geq c_{0} / 2$ or $\lambda^{k}-\mu^{k} \geq c_{0} / 2$. Let us assume that $\lambda^{k}+\mu^{k} \geq c_{0} / 2$. Then by the same proof as for Claim 5, we have the following.

Claim 6. Assuming that $\lambda^{k}+\mu^{k} \geq c_{0} / 2$, we have $\int_{I_{k}} u_{-1, k}^{2} d x \rightarrow \int_{\mathbb{R}} u_{-1}^{2} d x$ as $k \rightarrow \infty$.

Now it remains to show that $\int_{I_{k}} u_{-1, k}^{2} d x \rightarrow \int_{\mathbb{R}} u_{-1}^{2} d x$ as $k \rightarrow \infty$. Suppose this is not true. By Claim 3, we may assume that $\lim _{k \rightarrow+\infty}\left(\lambda^{k}-\mu^{k}\right)=0$. In fact, if $\lim _{k \rightarrow+\infty}\left(\lambda^{k}-\mu^{k}\right) \geq C>0$, then similar arguments to those in Claim 5 show that $u_{-1, k}$ has exponential decay and hence $\int_{I_{k}} u_{-1, k}^{2} d x \rightarrow \int_{\mathbb{R}} u_{-1}^{2} d x$ as $k \rightarrow \infty$, which contradicts our assumption.

Claim 7. $u_{1} u_{0}^{2} \equiv 0$ and $u_{-1} \equiv 0$.

Using $\int_{I_{k}} u_{-1, k}^{2} d x \rightarrow \int_{\mathbb{R}} u_{-1}^{2} d x$ as $k \rightarrow \infty$, we see that the limit $u_{-1}$ satisfies $\frac{1}{2} u_{-1}^{\prime \prime}-E_{-1} u_{-1}-\left(\beta_{n}+\beta_{s}\right) u_{-1}^{3}-\left(\left(\beta_{n}+\beta_{s}\right) u_{0}^{2}+\left(\beta_{n}-\beta_{s}\right) u_{1}^{2}\right) u_{-1}-\beta_{s} u_{0}^{2} u_{1}-B u_{0}=0$, in $\mathbb{R}$.

Integrating from 0 to $x$, we obtain that $\left|u_{-1}^{\prime}(x)\right| \geq C\left|\int_{0}^{x} u_{1} u_{0}^{2}\right|$. Since $\left\|u_{-1}\right\|_{H^{1}} \leq C$, we derive $u_{-1}=0$ and $u_{1} u_{0}^{2}=0$. If both $u_{1}=0$ and $u_{0}=0$, we then derive $N=-M$ (since $u_{1, k} \rightarrow u_{1}$ and $u_{0, k} \rightarrow u_{0}$ strongly in $\left.L^{2}(\mathbb{R})\right)$, which is impossible.

There are two cases to be considered.

Case 1. $u_{0}>0, u_{1}=0$.

By Claim 5, $\lambda^{k} \geq C>0$. Since $u_{j} \in H^{1}(\mathbb{R})$ and $u_{j}$ is decreasing, we see that $u_{j}(x) \rightarrow 0$ as $|x| \rightarrow+\infty$. Thus, for any $\delta>0$, we can find $R_{\delta}>0$ such that for $|x| \geq R_{\delta}$ 
we have $u_{j}(x) \leq \delta / 2$. As a consequence of the decreasing property of $u_{j, k}$, we can find $k_{0}$ such that $u_{j, k}(x) \leq u_{j, k}\left(R_{\delta}\right)<\delta$, for $|x|>R_{\delta}, k \geq k_{0}$. Then by $u_{1}=u_{-1}=0$, we see that $\left|\left(\beta_{n}+\beta_{s}\right) u_{1, k}^{2}+\left(\beta_{n}+\beta_{s}\right) u_{-1, k}^{2}+2 \beta_{s} u_{1, k} u_{-1, k}+B\left(u_{1, k}+u_{-1, k}\right)\right| \rightarrow 0$ uniformly in $\mathbb{R}$. Hence from the equation for $u_{0, k}$ and Claim $7, u_{0, k}$ satisfies

$$
\frac{1}{2} u_{0, k}^{\prime \prime}-E_{0} u_{0, k}-\beta_{n} u_{0, k}^{3} \geq \frac{c_{0}}{2} u_{0, k}, u_{0, k}>0 \quad \text { in }(-k, k), \quad u_{0, k}( \pm k)=0 .
$$

Using the equation for $u_{-1, k}$ and $\lim _{k \rightarrow+\infty}\left(\lambda^{k}-\mu^{k}\right)=0$, we see that $u_{-1, k}$ satisfies

$\frac{1}{2} u_{-1, k}^{\prime \prime}-E_{-1} u_{-1, k}-\beta_{n} u_{0, k}^{2} u_{-1, k} \leq \frac{c_{0}}{4} u_{-1, k}, u_{-1, k}>0 \quad$ in $(-k, k), \quad u_{-1, k}( \pm k)=0$.

Multiplying (3.4) by $u_{-1, k}$ and (3.5) by $u_{0, k}$ and then integrating over $(-k, k)$, since $E_{0}=E_{-1}$, we obtain a contradiction.

Case 2. $u_{1}>0, u_{2}=0$.

In this case, we observe that $u_{1}$ satisfies

$$
\frac{1}{2} u_{1}^{\prime \prime}-E_{-1} u_{-1}-\left(\beta_{n}+\beta_{s}\right) u_{1}^{3}=2 \lambda^{0} u_{1} \quad \text { in } \mathbb{R}, u_{1} \in H^{1}(\mathbb{R})
$$

where $\lim _{k \rightarrow+\infty} \lambda^{k}=\lim _{k \rightarrow+\infty} \mu^{k}=\lambda^{0}>0$.

On the other hand, $u_{0, k}(x) / u_{0, k}(0) \rightarrow \widehat{u}_{2}(x)$ which satisfies

$$
\frac{1}{2} \widehat{u}_{2}^{\prime \prime}-E_{0} \widehat{u}_{2}-\left(\beta_{n}+\beta_{s}\right) u_{1}^{2} \widehat{u}_{2}=\lambda^{0} \widehat{u}_{2} .
$$

It is easy to see that $0<\widehat{u}_{2} \leq 1$ since $\widehat{u}_{2}(0)=1$. Multiplying (3.6) by $\widehat{u}_{2}$ and (3.7) by $u_{1}$ and then integrating over $\mathbb{R}$, since $E_{0}=E_{-1}$, we get

$$
\lambda^{0} \int_{\mathbb{R}} u_{1} \widehat{u}_{2}=0
$$

which is impossible.

In conclusion, we have proved that as $k \rightarrow+\infty$ then $\int_{I_{k}} u_{j, k}^{2} \rightarrow \int_{\mathbb{R}} u_{j}^{2}$ for $j=-1,0,1$.

This completes the proof of Theorem 1.1.

\section{Characterisation of the ground state}

We denote the energy density $h$ by

$$
\begin{aligned}
h(\mathbf{u})=h\left(u_{-1}, u_{0}, u_{1}\right)=\sum_{j=-1}^{1}\left(\frac{1}{2}\left|u_{j}^{\prime}\right|^{2}+E_{j} u_{j}^{2}\right)+\frac{\beta_{n}}{2}\left(\sum_{j=-1}^{1} u_{j}^{2}\right)^{2} \\
+\frac{\beta_{s}}{2}\left(\left(u_{1}^{2}-u_{-1}^{2}\right)^{2}+2 u_{0}^{2}\left(u_{1}+u_{-1}\right)^{2}\right)+2 B u_{0}\left(u_{1}+u_{-1}\right) .
\end{aligned}
$$

Then

$$
\int_{\mathbb{R}} h(\mathbf{u}) d x=H(\mathbf{u}) .
$$


We also denote the set of all minimisers of $H$ (over $\mathcal{A}$ ) by $G$, where

$$
\mathcal{A}=\left\{\mathbf{u}=\left(u_{-1}, u_{0}, u_{1}\right) \in E_{N, M} \mid u_{j} \geq 0 \text { for } j=-1,0,1\right\} .
$$

The Euler-Lagrange equations for $\mathbf{u} \in G$ are given by the following coupled GrossPitaevskii equations:

$$
\left\{\begin{aligned}
\frac{1}{2} u_{-1}^{\prime \prime}-E_{-1} u_{-1}-\left(\beta_{n}+\beta_{s}\right) u_{-1}^{3}-\left(\left(\beta_{n}+\beta_{s}\right) u_{0}^{2}+\left(\beta_{n}-\beta_{s}\right) u_{1}^{2}\right) u_{-1}-\beta_{s} u_{0}^{2} u_{1}-B u_{0} \\
\quad=(\lambda-\mu) u_{-1} \\
\frac{1}{2} u_{0}^{\prime \prime}-E_{0} u_{0}-\beta_{n} u_{0}^{3}-\left(\beta_{n}+\beta_{s}\right)\left(u_{1}^{2}+u_{-1}^{2}\right) u_{0}-2 \beta_{s} u_{-1} u_{0} u_{1}-B\left(u_{1}+u_{-1}\right)=\lambda u_{0} \\
\frac{1}{2} u_{1}^{\prime \prime}-E_{1} u_{1}-\left(\beta_{n}+\beta_{s}\right) u_{1}^{3}-\left(\beta_{n}+\beta_{s}\right) u_{0}^{2} u_{1}-\left(\beta_{n}-\beta_{s}\right) u_{-1}^{2} u_{1}-\beta_{s} u_{0}^{2} u_{-1}-B u_{0} \\
\quad=(\lambda+\mu) u_{1}
\end{aligned}\right.
$$

where $\lambda$ and $\mu$ are the Lagrange multipliers.

Similar to the proof of Lemma 2.1 in [12], we give the following lemma.

Lemma 4.1. If $\mathbf{u} \in G \cap\left(C^{2}(\mathbb{R})\right)^{3}$, then for each $j$, either $u_{j}>0$ or $u_{j} \equiv 0$ in $\mathbb{R}$.

We recall some results on mass-redistribution of $n$-tuples of real-valued functions.

Definition 4.2 [12]. Let $\mathbf{f}=\left(f_{1}, f_{2}, \ldots, f_{n}\right) \in\left(H^{1}(\mathbb{R})\right)^{n}$ be an $n$-tuple of real-valued functions and $\mathbf{g}=\left(g_{1}, g_{2}, \ldots, g_{m}\right)$ be an $m$-tuple of nonnegative functions. We say $\mathbf{g}$ is a mass-redistribution of $\mathbf{f}$, if $g_{l}^{2}=\sum_{k=1}^{n} b_{l k} f_{k}^{2}$ for each $l$, where $b_{l k} \geq 0$ are constants and $\sum_{l=1}^{m} b_{l k}=1$ for each $k$.

We have the following proposition.

Proposition 4.3 [12]. For any mass-redistribution $\mathbf{g}$ of $\mathbf{f}$ as in Definition 4.2, we have the following results.

(1) $|\mathbf{g}|=|\mathbf{f}|$.

(2) $|\nabla g|^{2} \leq|\nabla \mathbf{f}|^{2}$. Moreover, $|\nabla g|^{2}=|\nabla \mathbf{f}|^{2}$ if and only if $f_{j} \nabla f_{k}=f_{k} \nabla f_{j}$ for each $j \neq k$ with $b_{l j} b_{l k} \neq 0$ for at least one $l$.

Our main result in this section is as follows.

THEOREM 4.4. Let $E_{-1}=E_{0}=E_{1}$ in the ferromagnetic system (1.1)-(1.3) for $n=1$, and let $\mathbf{u}=\left(u_{-1}, u_{0}, u_{1}\right) \in \mathcal{A}$ be the ground state of (1.1)-(1.3). Then we have the following results.

(i) If $\mathbf{u}$ satisfies $u_{0}\left(u_{-1}+u_{1}\right) \leq \sqrt{\frac{1}{2}\left(1-M^{2} / N^{2}\right)}|\mathbf{u}|^{2}$, then $h\left(\gamma^{*}|\mathbf{u}|\right) \leq h(\mathbf{u})$.

(ii) If $\mathbf{u} \in G \cap\left(C^{2}(\mathbf{R})\right)^{3}$ satisfies $u_{0}\left(u_{-1}+u_{1}\right)=\sqrt{\frac{1}{2}\left(1-M^{2} / N^{2}\right)}|\mathbf{u}|^{2}$, then $\mathbf{u}=\gamma^{*}|\mathbf{u}|$.

Here $\gamma^{*}=\left(\gamma_{-1}^{*}, \gamma_{0}^{*}, \gamma_{1}^{*}\right)$ is given by

$$
\gamma_{-1}^{*}=\frac{1}{2}\left(1-\frac{M}{N}\right), \quad \gamma_{0}^{*}=\sqrt{\frac{1}{2}\left(1-\frac{M^{2}}{N^{2}}\right)}, \quad \gamma_{1}^{*}=\frac{1}{2}\left(1+\frac{M}{N}\right) .
$$


Proof. By direct calculation,

$$
\begin{aligned}
h(\mathbf{u})-h\left(\gamma^{*}|\mathbf{u}|\right)=( & \left.|\nabla \mathbf{u}|^{2}-\left.|\nabla| \mathbf{u}\right|^{2}\right)-\beta_{s}\left(u_{0}^{2}-2 u_{-1} u_{1}\right)^{2} \\
& +2 B\left(u_{0}\left(u_{1}+u_{-1}\right)-\sqrt{\frac{1}{2}\left(1-\frac{M^{2}}{N^{2}}\right)}|\mathbf{u}|^{2}\right) .
\end{aligned}
$$

Then by Proposition 4.3 and $u_{0}\left(u_{-1}+u_{1}\right) \leq \sqrt{\frac{1}{2}\left(1-M^{2} / N^{2}\right)}|\mathbf{u}|^{2}, \beta_{s}<0$, we prove (i).

If $\mathbf{u} \in G$, from (i), we have $H(\mathbf{u})=H\left(\gamma^{*}|\mathbf{u}|\right)$, which in turn implies $h(\mathbf{u})=h\left(\gamma^{*}|\mathbf{u}|\right)$. Hence from (2) of Proposition 4.3,

$$
\begin{gathered}
f_{j} \nabla f_{k}=f_{k} \nabla f_{j} \quad \text { for } j \neq k ; \\
u_{0}^{2}=2 u_{-1} u_{1} ; \\
u_{0}\left(u_{1}+u_{-1}\right)=\sqrt{\frac{1}{2}\left(1-\frac{M^{2}}{N^{2}}\right)}|\mathbf{u}|^{2} .
\end{gathered}
$$

Now assume $\mathbf{u} \in G \cap\left(C^{2}(\mathbf{R})\right)^{3}$. From Lemma 4.1, at least one $u_{j}$ is strictly positive in $\mathbb{R}$. Without loss of generality assume $u_{1}>0$ in $\mathbb{R}$. Then from (4.1)

$$
\nabla\left(\frac{u_{0}}{u_{1}}\right)=\nabla\left(\frac{u_{-1}}{u_{1}}\right)=0 .
$$

Since $\mathbb{R}$ is connected, by (4.3), it follows that $u_{-1}$ and $u_{0}$ are both constant multiples of $u_{1}$. Hence (ii) follows by (4.2) and (4.3).

Remark. Theorem 4.4 implies that, under some condition, searching for the ground state of a ferromagnetic spin-1 BEC with an external Ioffe-Pitchard magnetic field can be reduced to a 'one-component' minimisation problem.

\section{References}

[1] M. H. Anderson, J. R. Ensher, M. R. Matthews, C. E. Wieman and E. A. Cornell, 'Observation of Bose-Einstein condensation in a dilute atomic vapor', Science 269 (1995), 198-201.

[2] W. Bao and F. Y. Lim, 'Computing ground states of spin-1 Bose-Einstein condensates by the normalized gradient flow', SIAM J. Sci. Comput. 30 (2008), 1925-1948.

[3] W. Bao and F. Y. Lim, 'Numerical methods for computing the ground state of spin-1 Bose-Einstein condensates in uniform magnetic field', Phys. Rev. E (3) 78 (2008), article 066704, 11 pages.

[4] C. C. Bradley, C. A. Sackett, J. J. Tollett and R. G. Hulet, 'Evidence of Bose-Einstein condensation in an atomic gas with attractive interaction', Phys. Rev. Lett. 75 (1995), 1687-1690.

[5] E. N. Bulgakov and A. F. Sadreev, 'Vortex phase diagram of $F=1$ spinor Bose-Einstein condensates', Phys. Rev. Lett. 90 (2003), 200401-200404.

[6] D. Cao, I.-L. Shern and J. Wei, 'On ground state of spinor Bose-Einstein condensates', NoDEA Nonlinear Differential Equations Appl. 18 (2011), 427-445.

[7] M. Chang, Q. Qin, W. Zhang, L. You and M. S. Chapman, 'Coherent spinor dynamics in a spin-1 Bose condensate', Nature Phys. 1 (2005), 111-116.

[8] K. B. Davis, M.-O. Mewes, M. R. Andrews, N. J. Vam Druten, D. S. Durfee, D. M. Kurn and W. Ketterle, 'Bose-Einstein condensation in a gas of sodium atoms', Phys. Rev. Lett. 75 (1995), 3969-3973. 
[9] E. P. Gross, 'Structure of a quantized vortex in boson systems', Nuovo Cimento X. Ser. 20 (1961), 454-477.

[10] T. L. Ho and V. B. Shenoy, 'Binary mixtures of Bose condensates of alkali atoms', Phys. Rev. Lett. 77 (1996), 3276-3279.

[11] T. Isoshima, K. Machida and T. Ohmi, 'Spin-domain formation in spinor Bose-Einstein condensation', Phys. Rev. A (3) 60 (1999), 4857-4863.

[12] L. Lin and I.-L. Shern, 'Characterization of the ground states of spin-1 Bose-Einstein condensates', arXiv:1102.0832v2, 2011.

[13] H. J. Miesner, D. M. Stamper-Kurn, J. Stenger, S. Inouye, A. P. Chikkatur and W. Ketterle, 'Observation of metatable states in spinor Bose-Einstein condensates', Phys. Rev. Lett. 82 (1999), 2228-2231.

[14] T. Ohmi and K. Machida, 'Bose-Einstein condensation with internal degrees of free- dom in alkali atom gases', J. Phys. Soc. Japan 67 (1998), 1822-1825.

[15] L. P. Pitaevskii, 'Vortex lines in an imperfect Bose gas', Soviet Phys. JETP 13 (1961), 451-454.

[16] L. P. Pitaevskii and S. Stringari, Bose-Einstein Condensation (Clarendon Press, Oxford, 2003).

[17] J. Stenger, S. Inouye, D. M. Stamper-Kurn, H. J. Miesner, A. P. Chikkatur and W. Ketterle, 'Spin domians in ground-states Bose-Einstein condensates', Nature London 396 (1998), 345-348.

WEI LUO, School of Mathematical Sciences, Xuzhou Normal University, Xuzhou, 221116, PR China

ZHONGXUE LÜ, School of Mathematical Sciences, Xuzhou Normal University, Xuzhou, 221116, PR China

e-mail: lvzx1@tom.com

ZUHAN LIU, School of Mathematical Sciences, Xuzhou Normal University, Xuzhou, 221116, PR China 